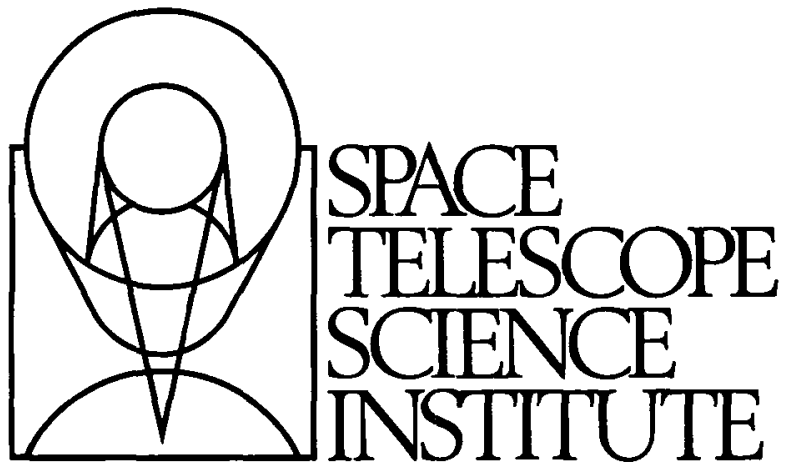

NASA-CR-183,251

NASA-CR-183251

19880021171

PREPRINT SERIES

No.205

\title{
OBSERVATIONS OF FAINT FIELD GALAXIES
}

David C. Koo

(AASA-CR-183251) OESEEVATICASCE FAINT

FIELD GAIAXIES (SFace TeJESCCFE science

Inst.) \& $\mathrm{F}$
$\operatorname{CSCL} 03 \mathrm{~A}$
N88-30555

Unclas

G3/89 $01619 \equiv 1$

September 1987

SPACE TELESCOPE SCIENCE INSTITUTE

3700 San Martın Drive Baltımore, MD 21218 


\section{OBSERVATIONS OP FAINT FIELD GALAXIES \\ David C. Koo \\ Space Telescope Science Institute \\ 3700 San Martin Drive \\ Baltimore, MD 21218}

To be published in the proceedings of the

I.A.U. Symposium \#130

"Evolution of Large Scale Structures in the Universe"

eds. J. Audouze and A. S. Szalay (Reidel, Dordrecht) 
OBSERVATIONS OF FAINT FIELD GALAXIES

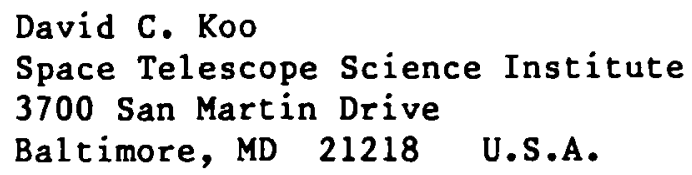

ABSTRACT. Number counts, colors, and angular correlations of field galaxies fainter than 20 th mag are summarized. Resulting conclusions regarding the presence and nature of luminosity, spectral, and clustering evolution remain controversial. Preliminary analysis of two major spectroscopic surveys near completion suggests that by $z \sim 0.5$, larger numbers of very blue galaxies of moderate luminosities are found than today. These skewer-like surveys also provide new probes of galaxy clustering on scales previously unexplored ( $>200 \mathrm{Mpc}$ ) and over lookback times of several billion years.

\section{INTRODUCTION}

Over the last decade, major progress has been made in studying the properties of field galaxies with $B>20$, faint enough to hope that evolution would be measureable. These new observations have included deep number counts, average color changes with magnitude, angular correlation functions, and even rough redshifts based upon multicolor photometry. Progress is taking another leap with the availability of multiaperture CCD spectroscopy. Hundreds of redshifts of typical galaxies at $z>0.1$ is no longer a dream and thus the content and structure of the universe billions of years in the past is now explorable. This talk is largely a progress report on the status of two such redshift surveys, one made at Kitt Peak National Observatory and the other at the Anglo-Australian Observatory, and both with $4 \mathrm{~m}$ telescopes. The exciting work from the Soviet $6 \mathrm{~m}$ telescope is reported by Shvartsman and Karachentsev.

\section{SUMMARY OF PHOTOMETRIC SURVEYS}

\subsection{Counts and Colors of Faint Field Galaxies}

Number counts of galaxies have been extended by nearly a factor of 1000 in faintness in the last ten years. The first major step was 
the result of combining the sensitivity and dynamic range of new photographic emulsions (hypersensitized by baking) with the fast prime focus cameras of new $4 \mathrm{~m}$ class telescopes. These surveys reached B $\sim 24$, well past the B $\sim 2111 \mathrm{mits}$ of data from the previous generation and plenty deep to study evolution and cosmology. The major conclusion is that more faint galaxies are observed at fainter magnitudes than expected, if the properties of distant galaxies were on average the same as that today (E11is 1982, Shanks et al. 1984). In fact, number counts of galaxies from bluer passbands appear to rise more steeply with magnitude, with the ultraviolet counts increasing at a Euclidian rate of 0.6 in dex-mag versus 0.4 with the $R$ and I bands (Koo 1986). This is consistent with the other major conclusion of these photometric surveys, that faint galaxies appear quite blue, first reported by Kron $(1978,1980)$. Both the steep counts and blue colors can be understood in the context of galaxy evolution, whereby distant galaxies were brighter and bluer on average than today due to more extensive star formation (see e.g. models by Tinsley 1980 or Bruzual and Kron 1980); the best-fitting models generally adopt a cosmology requiring a low density universe and a high redshift $(z>5)$ for the assumed single epoch of galaxy formation.

Despite a common belief that evolution has indeed been detected, doubts legitimately remain, since counts from different groups vary by as much as a factor of two (same size as the difference between models with and without evolution) and the conclusions depend on relatively poor calibrations of the local luminosity functions and UV spectra of galaxies of different types (or colors). Other possible problems include photometric zero points, galactic extinction, different methods of photometry, and even large-scale clustering. With CCD's that now achieve an impressive gain of several more magnitudes to $B=27$ (Tyson 1987) or $R=25.7$ (Hall and Mackay 1984), the trends in both counts and colors are confirmed, thus strengthening the conclusions from the brighter photographic surveys; in addition, the count variations from field to field are claimed to be smal1, about $10 \%$ or twice Poisson, for the CCD data (Tyson 1987), a point to which we will later return.

\subsection{Angular Correlations}

Besides counts and colors, angular correlations can be measured from essentially the same data and provide a probe of clustering evolution. As with the counts, the results vary significantly among the groups who have used $4 \mathrm{~m}$ plates (see Ellis 1987 for a review). At the high end, the data fit a model that assumes no or mild luminosity evolution, is consistent with the counts and colors, and includes no evolution in the clustering; at the other end, the data gives evidence for some growth in clustering over time. Besides the uncertainties that plague interpretations of counts and colors, an accurate analysis also needs to account for (but have so far not) the known differences in the clustering properties (slope and amplitude of the power-law correlation) of different types of galaxies (Davis 
and Geller 1976, Giovanelli et al. 1986); any features, such as the "shoulder", in the spatial correlation function (Shanks et al. 1983) would only further complicate the modeling.

\section{SUMMARY OF DEEP REDSHIFT SURVEYS}

\subsection{Redshifts Based On Multicolor Photometry}

Redshifts, when combined with photometry, are essential for reliable measurements of intrinsic parameters, such as luminosity and color, of faint galaxies. One technique that has the advantage of reaching very faint limits is to rely on broad or intermediate band photometry to estimate rough redshifts and galaxy type by matching the observed spectral energy distributions (SED) of faint objects with a set of redshifted templates. Although there are problems associated with accurate calibrations and measurements of systematic and random errors (see E11is 1987), the method has promise. For example, Couch et al. (1983), Ellis et al. (1985), and MacLaren et al. (1987) have used intermediate band filters and CCDs to study the SEDs of galaxies in distant clusters and find that red galaxies possess a wide dispersion of flux in the ultraviolet; Loh and Spillar (1986a, 1986b) have adopted a similar setup on a sample of 1000 faint field galaxies to conclude that we $l$ ive in a high density universe (i.e. $\Omega=1$ ) and that little color or luminosity evolution has occurred up to $z \sim 0.8$; and Koo (1986) has employed broadband UBVI photography of faint field galaxies to find that the fraction of intrinsically blue ( $B-V<0.7)$ galaxies of moderate luminosity has increased to $~ 65 \%$ at $z \sim 0.4$ from the $~ 45 \%$ found today. Loh (this conference) has even exploited the method to measure the clustering of $z \sim 0.5$ field galaxies and concludes that growth of clustering has occurred over the last few billion years, a result that supports the low side of the conflicting angular correlation observations.

\subsection{Spectroscopic Observations}

There is no substitute for spectroscopic redshifts; even methods based on multicolor photometry must rely on such data for proper calibration. With the availability of CCDs and multiaperture spectrograph systems, redshifts of galaxies fainter than $B=20$ can be routinely acquired now, though the effort is still difficult and requires many hours on a large telescope. Perhaps the most dramatic results have been reported for the study of distant clusters (see talk by Dressler). Redshift surveys of field galaxies, though initiated around 1981, have only recently been complete enough to yield some preliminary results (E1Lis 1987, Koo and Kron 1987). A summary of the observations are given in Table 1. 
Table 1. Deep Redshift Surveys of Field Galaxies

\begin{tabular}{|c|c|c|}
\hline & $\begin{array}{c}\text { KPNO } \\
\text { (Koo and Kron) }\end{array}$ & $\begin{array}{c}\text { AAT } \\
\text { (Ellis, Broadhurst, and Shanks) }\end{array}$ \\
\hline $\begin{array}{l}\text { Telescope } \\
\text { Instrument- } \\
\text { Detector } \\
\text { No. of fields } \\
\text { Field Size } \\
\text { Apertures } \\
\text { Range ( } \AA \text { ) } \\
\text { Resolution ( }(\AA) \\
\text { Exposure (hr) } \\
\text { Mag Limit } \\
\text { Completeness ( }() \\
\text { \# of redshifts } \\
\text { Photometry }\end{array}$ & $\begin{array}{l}4.0 \mathrm{~m} \\
\text { Cryogenic Camera } \\
\quad+\mathrm{TI} \text { CCD } \\
3+ \\
40^{\prime} \text { diameter } \\
40 \text { holes or } 10 \text { slits } \\
4500-7500 \\
15 \\
1-3 \\
\text { R } 21 \\
100(\mathrm{R}<19) \text { to } \\
6(20.5-21) \\
400+ \\
\text { Photog. UBVI }\end{array}$ & $\begin{array}{l}3.9 \mathrm{~m} \\
\text { RGO / Faint Obj Red Spec } \\
+ \text { IPCS / + GEC CCD } \\
5 \\
20^{\prime} \text { diameter } \\
50 / 50 \text { fibers } \\
3700-6100 / 5000-10,000 \\
4 / 15 \\
4-6 \\
20<\text { B } \\
85(B<21.5 \\
75(B<21.5) \\
200+ \\
\text { most1y Photog. B }\end{array}$ \\
\hline
\end{tabular}

\subsection{Results on Galaxy Evolution}

The following summary is based upon the reports of Ellis (1987) for the AAT survey and of Koo and Kron $(1987,1988)$ for the KPNO survey. To the surprise of both groups, the overall redshift distributions are most consistent with no or little luminosity evolution in galaxies, i.e. too few luminous galaxies are found at high redshifts $z>0.5$. Although the AAT survey remains incomplete, [OII] $3727 \AA$ is easily observable to $z<1$, and so the lack of high redshifts is probably real; $85 \%$ of the current sample shows emission lines. For the KPNO work, completeness is not an issue, since a subsample of 300 galaxies has completeness that is well defined but varies with magnitude.

To explain the excess of counts, both teams also concur that galaxies with more extensive star formation are found at redshifts $z$ $>0.25$ than today. The AAT group bases this conclusion on finding that many such galaxies have [OII] rest-frame equivalent widths larger than $20 \AA$, but unlike local counterparts which tend to be Im dwarfs of low luminosity, these high redshift blue galaxies are as bright as typical spirals. They also argue that neither the aperture effect nor $K$-corrections are significant selection effects. In contrast, the KPNO conclusion is based directly on finding twice as many $z>0.25$ galaxies with the UV-bright colors of Im type galaxies than predicted by no-evolution models. Finally, neither survey yield large numbers of low-luminosity dwarfs at low redshifts that might contribute to the excess of faint galaxies. 


\subsection{Results on Galaxy Clustering}

The narrowness of the conical volumes probed by the deep redshift surveys is worth emphasizing. At $z=0.3$, the 40 arcmin diameter fields of the KPNO surveys span about 6.5 Mpc (assuming $H_{0}=100 \mathrm{~km}$ $\mathrm{sec}^{-1} \mathrm{Mpc}^{-1}$ and $\left.\mathrm{q}_{0}=0.5\right)$. The AAT fields are half of this, but both surveys cover areas larger than the Mpc size of even rich clusters of galaxies. Superclusters of several tens of Mpc, however, may only be partially intercepted.

As reported by E11is (1987) for the AAT work, their survey yields a spatial correlation function which is "tantalisingly lower than expected in the virialised case but about twice the number of galaxies is needed before any significance can be attached to this result." Moreover, they identify "sheet-1ike structures" in some of the cones and find that the absolute number of galaxies counted to deeper limits is well correlated with the number of such sheets.

As reported by Koo, Kron, and Szalay (1987) for the KPNO survey, a major surprise is the strong clumpiness seen in the redshift distributions, especially for the SA 57 field (Figure la). Some redshift spikes contain rich groups or even a cluster (e.g. note $z=$ 0.12 and 0.24 clumps in Fig. 2a), but others do not (e.g. at $z=0.3$ in Fig. 2b). The smooth distributions are consistent with flattened large scale structures, i.e. sheets or pancakes being skewered by our observing cones. These structures, whatever their shape, can strongly affect the counts of galaxies; e.g., over $50 \%$ of all the B < 20 galaxies in a $0.3 \mathrm{deg}^{2}$ area in SA 57 occupies the single peak at $z$ $=0.12$. Very deep CCD frames, on the other hand, penetrate a larger number of such structures and should thus experience far less fluctuations from field to field.

It is interesting to note, however, that the observed fluctuations are already quite consistent with extrapolations of the simple power-law clustering of the B 15 Zwicky sample, so perhaps no excess at large scales is needed. The ratio, $R$, of expected fluctuations to that from Poisson (assuming the angular correlation amplitudes scale as $\mathrm{N}^{-0.6}$ ) is:

$$
R \sim\left[1+(N S)^{0.4}\right]^{0.5}
$$

where $N$ is the number of galaxies observed in a box with sides, $S$, in degrees (Phillipps and Disney 1985). In the case of $1^{\circ} \times 1^{\circ}$ fields, 4m plates yield $\sim 3500$ galaxies for $20<B<22.5$, so $R$ should be about 5; this matches the results reported by Ellis (1987). In the case of a typical CCD field of $3 \times 3$ arcmin with, e.g., 400 galaxies that might reach $B$ about $25, R$ should be about 2 ; this is as observed by Tyson (1987).

Another feature of interest is the "void" of galaxies centered at $z \sim 0.15$ in the SA 57 data, where one galaxy is detected from an expected 15. To check if this gap of over $100 \mathrm{Mpc}$ in depth extends across in the plane of the sky, we have searched for all Abell clusters that have spectroscopic redshifts and that are less than 15 deg from our field. A total of 56 are found, 37 with $z<0.13,18$ 


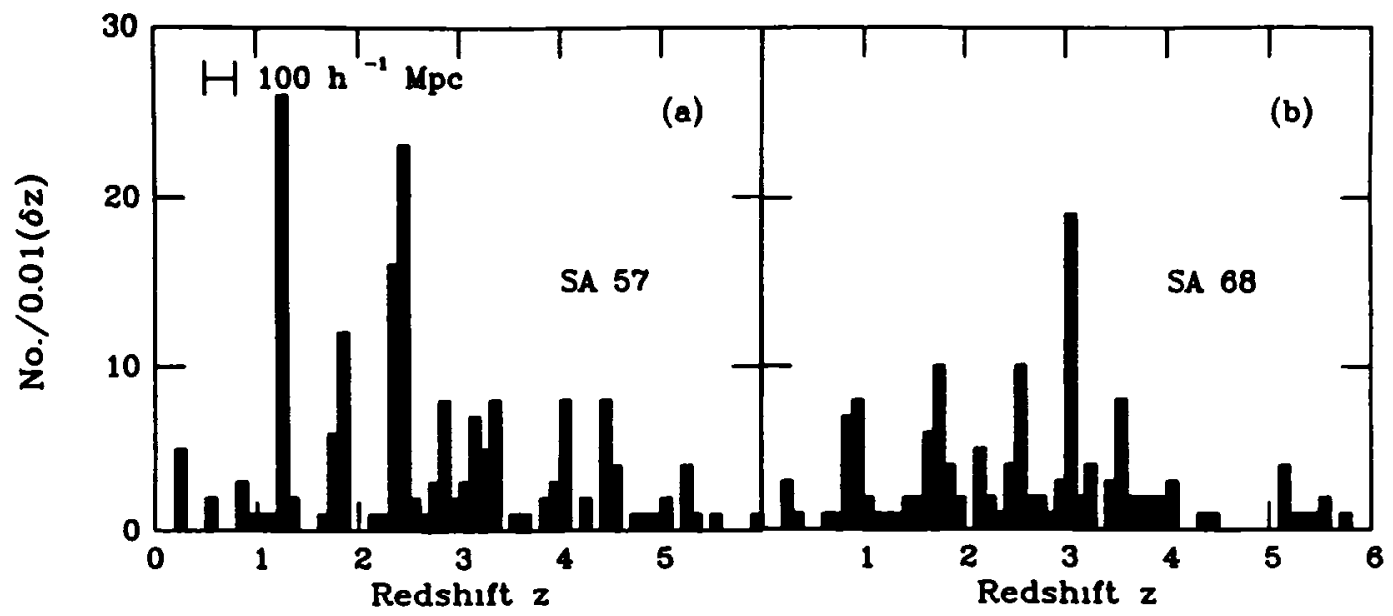

Figure 1. a) Histogram in bins of 0.01 in $z$ of redshifts for 148 galaxies within a $0.3 \mathrm{deg}^{2}$ field at the North Galactic Pole (SA 57). The magnitude and completeness 1 imits are complex but with most galaxies having $20<B<23$. b) Histogram of 134 redshifts in SA 68 .

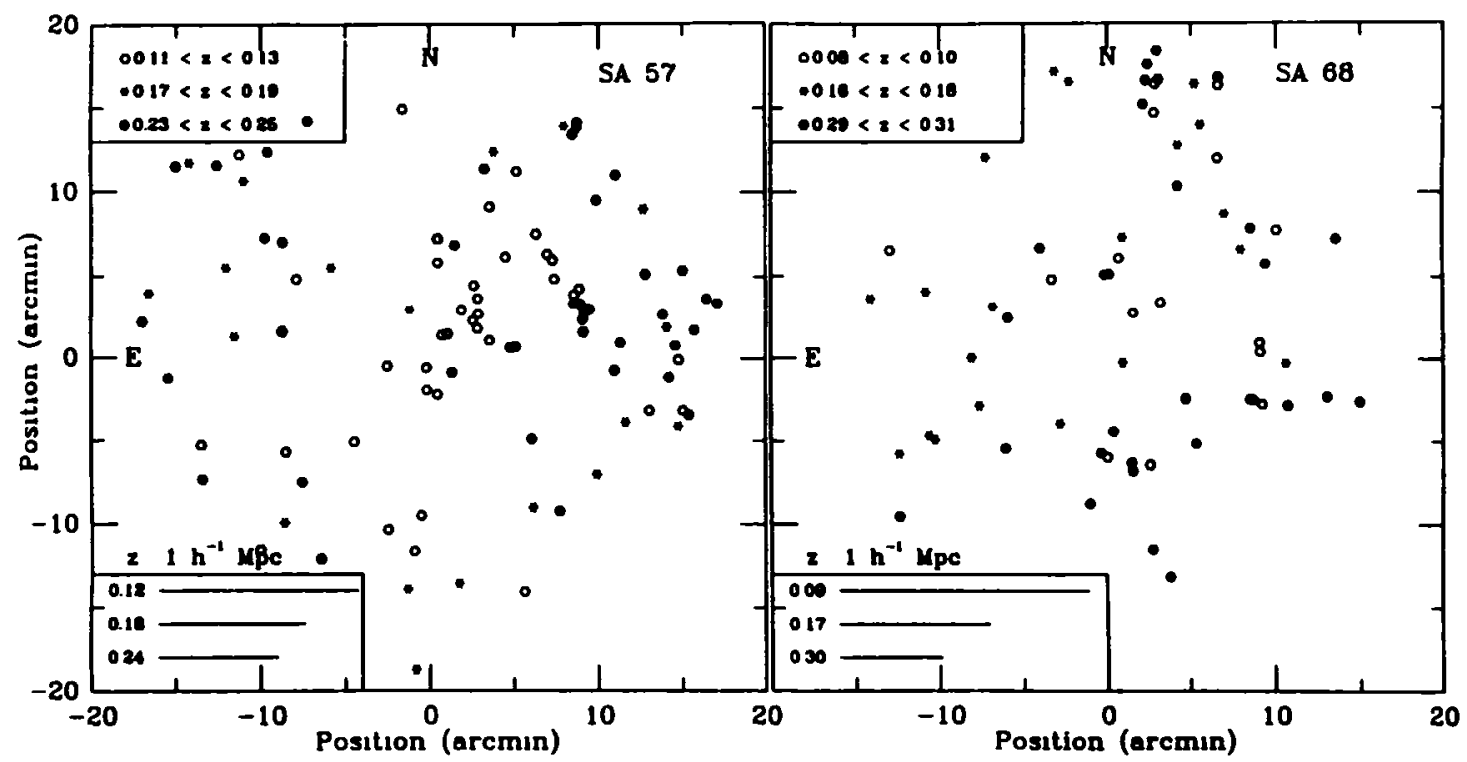

Figure 2. a) Sky positions of SA 57 galaxies with redshifts near the peaks at $z=0.12,0.18$, and 0.24 . Coverage of the field is NOT uniform. b) Same for SA 68 galaxies at $z=0.09,0.17$, and 0.30 .

with $z>0.16$, and one at $z=0.14$, which is in the gap, but this cluster is at the edge of our search area almost $100 \mathrm{Mpc}$ away! On the other hand, $\mathrm{N}$-body simulations of deep redshift surveys by White et al. (1987) of a universe with cold dark matter, biased galaxy formation, and $\Omega=1$ also show spiky distributions, so we should perhaps reserve judgement on the meaning of our redshift distribu- 
tions until more detailed comparisons are made. The redshift data is still being improved in reliability, completeness, and accuracy. When completed and combined with multicolor photometry, this and other surveys promise to provide powerful tools to study evolution of the spectral and clustering properties of distant field galaxies.

I thank R. S. Ellis for helpful discussions. Figure 2 shows unpublished data from a joint project with R. Kron and A. Szalay.

\section{REFERENCES}

Bruzual, G. A. and Kron, R. G. 1980 Ap. J., 241, 25.

Couch, W. J., Ellis, R. S., Godwin, J., and Carter, D. 1983 M.N.R.A.S., 205, 1287.

Davis, M. and Geller, M. J. 1976 Ap. J., 208, 13.

Ellis, R. S. 1982 in Origin and Evolution of Galaxies, eds. B. J. T. Jones and J. E. Jones (Reidel, Dordrecht) p. 255.

Ellis, R. S. 1987 in IAU No. 124, Observational Cosmology, eds. A. Hewitt, G. Burbidge, and L. Z. Fang (Reidel, Dordrecht) p. 367.

Ellis, R. S., Couch, W. J., MacLaren, I., and Koo, D. C. 1985 M.N.R.A.S., $217,239$.

Giovanelli, R., Haynes, M. P., and Chincarini, G. L. 1986 Ap. J., 300, 77.

Hal1, P. and Mackay, C. D. 1984 M.N.R.A.S., $210,979$.

Koo, D. C. 1986 Ap. J., 311, 651.

Koo, D. C. and Kron, R. G. 1987 in IAU No. 124, Observational Cosmology, eds. A. Hewitt, G. Burbidge, and L. Z. Fang (Reidel, Dordrecht) p. 383 .

- 1988 in Towards Understanding Galaxies at Large Redshifts, eds. R. G. Kron and A. Renzini (Reidel, Dordrecht).

Koo, D. C., Kron, R. G., and Szalay, A. S. 1987 in Proceedings of 13th Texas Symposium on Relativistic Astrophysics, ed. M. P. Ulmer (World Scientific, Singapore) p. 284.

Kron, R. G. $1978 \mathrm{Ph}$. D thesis, University of California, Berkeley. - 1980 Ap. J. Suppl., 43, 305.

Loh, E. D. and Spillar, E. J. 1986a Ap. J., 303, 154. - 1986 b Ap. J. (Lett), 307, L1.

MacLaren, I, E1lis, R. S., and Couch, W. J. 1987 M.N.R.A.S., in press.

Phillipps, S. and Disney, M. J. 1985 A. A., 148, 234.

Shanks, T., Bean, A. J., Efstathiou, G., Ellis, R. S., Fong, R., and Peterson, B. A. 1983 Ap. J., 274, 279.

Shanks, T., Stevenson, P. R. F.,Fong, R., and MacGillivray, H. T. 1984 M.N.R.A.S., 206, 767.

Tinsley, B. M. 1980 Ap. J., 241, 41.

Tyson, J. A. 1987 , preprint

White, S. D. M., Frenk, C. S., Davis, M., and Efstathiou, G. 1987 Ap. J., 313, 505. 
End of Document 